\title{
A GENERALIZATION OF A THEOREM OF JACOBSON
}

\section{SUSAN MONTGOMERY}

Abstract. A well-known theorem of Jacobson asserts that a ring $R$ in which $x^{n(x)}=x$ for each $x$ in $R$ must be commutative. This paper gives a description of a ring with involution in which the above condition is imposed only on the symmetric elements. In particular, if $R$ is primitive, $R$ is either commutative or the $2 \times 2$ matrices over a field, and, in general, any such $R$ is locally finite and satisfies a polynomial identity of degree 8 .

A well-known theorem of Jacobson asserts that a ring $R$ in which $x^{n(x)}=x$ for each $x$ in $R, n(x)$ an integer greater than 1 , must be commutative $[5$, p. 217]. In this paper we answer the following question: What can be said about a ring with involution in which the above condition is imposed only on the symmetric elements? If $R$ is a division ring, then $I$. N. Herstein and the author have shown that the ring still must be commutative [4]. However, as was pointed out in [4], it is not difficult to see that, in general, such a ring need not be commutative. For, consider the $2 \times 2$ matrices over a finite field of characteristic not 2 under the involution

$$
\left(\begin{array}{ll}
\alpha & \beta \\
\gamma & \delta
\end{array}\right)^{*}=\left(\begin{array}{rr}
\delta & -\beta \\
-\gamma & \alpha
\end{array}\right) .
$$

The ring is certainly not commutative, but the symmetric elements satisfy $s^{n(s)}=s$ for all symmetric elements $s$. We show that this example (or other examples built up from $2 \times 2$ matrix rings) is basically the only alternative to the algebra being commutative, in the case that the characteristic is not 2 .

The proofs depend heavily on the following results of J. M. Osborn [9]; it is interesting to note that in his proofs Osborn needed the result of I. N. Herstein and the author on division rings [4].

Definition. Let $A$ be a power-associative ring. Then $A$ is periodic if $x^{n(x)}=x$ for all $x \in A$.

Proposition (OsBoRN). Let $\Phi$ be a subfield of the algebraic closure of the integers mod $p$ for some $p \neq 2$, and let $\mu$ be a nonsquare in $\Phi$. Let $\Phi_{2}$ denote the ring of $2 \times 2$ matrices over $\Phi, \Phi_{2}^{+}$denote the same set under the

Received by the editors July $13,1970$.

AMS 1969 subject classifications. Primary 1658, 1650, 1642, 1649.

Key words and phrases. Rings with involution, commutativity, polynomial identity, algebraic algebras. 
Jordan product $x \circ y=\frac{1}{2}(x y+y x)$. Then the Jordan subalgebra of $\Phi_{2}^{+}$ consisting of the set

$$
J=\left\{\left(\begin{array}{cc}
\alpha & \beta \mu \\
\beta & \gamma
\end{array}\right) \mid \alpha, \beta, \gamma \in \Phi\right\}
$$

is a simple Jordan periodic ring of capacity 2. Conversely, every simple Jordan periodic ring of capacity 2 and characteristic not 2 is isomorphic to such an algebra $J$ for some choice of $\Phi$.

TheOREM (Osborn). A simple Jordan periodic ring of characteristic not 2 is either a field or a Jordan ring of capacity 2. Any Jordan periodic ring of characteristic not 2 is a subdirect sum of simple Jordan periodic rings.

We also use a theorem of Martindale [6] concerning Jordan homomorphisms:

TheOREM (MARTINDALE). Let $A$ be a ring with involution such that

(1) A contains 2 nonzero orthogonal symmetric idempotents $e_{1}, e_{2}$ whose sum is 1 ;

(2) $A_{i j} A_{j i}=A_{i i}, i=1,2$, where $A_{i j}=e_{i} A e_{j}$;

(3) $A_{i i}$ equals the associative subring generated by $S_{i}=S \cap A_{i i}, i=1,2$. Let $\phi$ be a Jordan homomorphism of the symmetric elements $S$ of $A$ into an arbitrary ring $R$ whose characteristic is not 2. Then $\phi$ can be extended uniquely to an associative homomorphism of $A$ into $R$.

The symmetric elements of a ring $R$ with an involution * will be denoted by $S$.

The first lemma establishes a simple identity.

Lemma 1. If $R$ is a ring of characteristic not 2, then the product $(a b-b a)^{2}=[a, b]^{2}$ may be written in terms of the Jordan product $x \circ y=\frac{1}{2}(x y+y x)$.

Proof. It is not difficult to verify that

$$
[a, b]^{2}=4(a \circ b)^{2}-4 a \circ\left(b^{2} \circ a\right)-4 b \circ\left(a^{2} \circ b\right)+4\left(a^{2} \circ b^{2}\right) \text {. }
$$

We are now able to completely describe the case of a simple ring which is finite-dimensional over its center.

Lemma 2. If $R$ is a simple ring with * of characteristic not 2, finitedimensional over its center $Z$, and such that $s^{n(s)}=s$ for all $s \in S$, then $R$ is a field or a $2 \times 2$ matrix ring over a field of characteristic $p \neq 0$.

Proof. We first show that $\operatorname{dim}_{z} R \leqq 4$. Assume that $\operatorname{dim}_{Z} R>4$. Then by a theorem of Herstein $[3$, p. 10], the subring generated by $S$ is all 
of $R$. It is also true that $S$ is a simple Jordan ring [3, pp. 27, 32]. Since $S$ is periodic, by Osborn's results either $S$ is a field or $S \simeq J$ as described in the proposition.

First assume that $S \simeq J \subseteq \Phi_{2}^{+}$. Then using $A=\Phi_{2}$ with $e_{1}=\left(\begin{array}{ll}1 & 0 \\ 0 & 0\end{array}\right)$ and $e_{2}=\left(\begin{array}{ll}0 & 0 \\ 0 & 1\end{array}\right)$, we see that $A$ satisfies the hypotheses of Martindale's theorem. Thus, the Jordan isomorphism $\phi: J \rightarrow S$ may be extended to an associative homomorphism $\phi_{1}$ of $\Phi_{2}$ into $R$. Since $\Phi_{2}$ is simple, $\phi_{1}$ is one-to-one. But since $S$ generates $R, \phi_{1}$ is also onto. Thus, $R \simeq \Phi_{2}$, so $\operatorname{dim}_{Z} R \leqq 4$, a contradiction.

Now say that $S$ is a field. Let $e$ be the unit for $S$; we show that in fact $e$ is a unit for $R$. For any $s \in S, s \circ e=\frac{1}{2}(s e+e s)=s$; that is, $s e+e s=2 s$, all $s \in S$. Thus,

$$
(s e-e s) e+e(e s-s e)=2(s e-e s) .
$$

Simplifying, this yields $s e=e s$. But then we get $2 e s=2 s=2 s e$, and so $s e=s=e s$. Since $S$ generates $R, e$ is the unit for $R$.

We are now able to show that every nonzero symmetric element is invertible in $R$. For, choose $s \neq 0$ in $S$ and let $r$ be its Jordan inverse in $S$. Thus, $r \circ s=s \circ r=e$. We know that $s^{n}=s$, for some $n$; since $S$ is a field, $o$ is associative and thus,

$$
e=s \circ r=s^{n} \circ r=\left(s^{n-1} \circ s\right) \circ r=s^{n-1} \circ(s \circ r)=s^{n-1} \circ e=s^{n-1} \text {. }
$$

Thus, $s$ is invertible. By a theorem of Osborn [8], if $R$ is a simple ring with involution of characteristic not 2 such that every nonzero symmetric element is invertible, then either $R$ is a division ring or $\operatorname{dim}_{Z} R \leqq 4$. If $R$ is a division ring, $R$ must be commutative [4] and so $R=Z$. In either case, $\operatorname{dim}_{Z} R \leqq 4$, and we have a contradiction. Thus, $\operatorname{dim}_{z} R \leqq 4$, and so $R$ must be a field or the $2 \times 2$ matrices over a field.

We can now characterize a primitive ring satisfying our condition.

THEOREM 1. If $R$ is a primitive ring with involution of characteristic not 2 such that $s^{n(s)}=s$ for all $s \in S$, then $R$ is a field of finite characteristic or the $2 \times 2$ matrices over such a field.

Proof. As in Lemma $2, S$ is a periodic Jordan ring. Thus, by Osborn's theorem, $S$ is a subdirect sum of fields and periodic Jordan rings of capacity 2 . Let $S_{\alpha}$ be any summand.

We claim that in each $S_{\alpha}$, the elements satisfy the identity $\left[[a, b]^{2}, c\right]^{2}=0$, all $a, b, c \in S_{\alpha}$ (by Lemma 1 this identity may be expressed in terms of the Jordan product). This is trivial if $S_{\alpha}$ is a field, so assume that $S_{\alpha}$ has capacity 2 ; by Osborn's proposition $S_{\alpha}$ consists of the symmetric elements in a $2 \times 2$ matrix ring. If $a$ and $b$ are any two matrices in $S_{\alpha}$, it is easy to see that $(a b-b a)^{2}$ is in the center of $S_{\alpha} ;$ thus, $\left[[a, b]^{2}, c\right]^{2}=0$ for any $c \in S_{\alpha}$. 
Since $S$ is a subdirect sum of the $S_{\alpha}$, we have that $\left[[a, b]^{2}, c\right]^{2}=0$ for all $a, b, c \in S$. This means that $S$ satisfies a polynomial identity. From results of Herstein [2] and Martindale [7], the entire ring $R$ must satisfy a polynomial identity. Since $R$ is primitive, $R$ must be simple and finite-dimensional over its center by a well-known result of Kaplansky [5, p. 226]. The theorem is now proved by applying Lemma 2.

We now proceed to the semisimple case. However, it is now necessary to assume, in addition, that $R$ is an algebra.

Lemma 3. If $R$ is a semisimple algebra with involution over a field $F$ of characteristic not 2 such that $s^{n(s)}=s$ for all $s \in S$, then $R$ is a subdirect sum of fields and $2 \times 2$ matrix rings over fields of finite characteristic.

Proof. Since $R$ is a subdirect sum of its primitive images, it will be enough to show that every primitive image of $R$ is a field or a $2 \times 2$ matrix ring. Let $P$ be a primitive ideal of $R$.

Now if $P^{*} \subseteq P$, then $R / P$ has an induced involution and so is a primitive ring satisfying the hypotheses of Theorem 1 . Thus, $R / P$ is a field or a $2 \times 2$ matrix ring.

If $P^{*} \Phi P$, then $P+P^{*} / P$ is a nonzero two-sided ideal of $R / P$, so is itself primitive with the same vector space and commuting ring as $R / P$. Choose $x \in P+P^{*} ; x=a+b$, where $a \in P, b \in P^{*}$. Thus, $b^{*} \in P$. Now $b+b^{*}=x+\left(b^{*}-a\right) \equiv x(\bmod P)$. Thus, every element $\bar{x}$ of $P+P^{*} / P$ is the image of a symmetric element of $R$, and so satisfies $\bar{x}^{n(\tilde{x})}=\bar{x}$. By Jacobson's theorem $P+P^{*} / P$ is commutative, and thus is a field. By looking at the action of $R / P$ and $P+P^{*} / P$, we see that $R / P$ is also a field.

Lemma 4. Let $R$ be an algebra with * over a field of characteristic not 2 such that $s^{n(s)}=s$, all $s \in S$. If $J(R)$ is the radical of $R$, then

(1) $J(R)^{3}=(0)$;

(2) $x \in J(R)$ implies $x^{2}=0$.

Proof. First of all, $S \cap J(R)=0$. For if $s \in S \cap J(R), s^{n}=s$, and thus $s^{n-1}$ is an idempotent in $J(R)$, which is impossible. Thus, if $x \in J(R)$, since $x+x^{*} \in J(R) \cap S$, it must be that $x+x^{*}=0$. That is, every element in $J(R)$ is skew. Since the square of a skew element is symmetric, (2) follows.

To show (1), we next see that for $x, y \in J(R), x y=-y x$; for, $(x+y)^{2}=0=x^{2}+x y+y x+y^{2}=x y+y x$. But then $J(R)^{3}=(0)$ follows easily; if $x, y, z \in J(R),(x y) z=-y x z=y z x=-x y z$. Since the characteristic is not $2, x y z=0$.

We now combine Lemmas 3 and 4 in the final theorem. 
THEOREM 2. Let $R$ be an algebra with involution over a field of characteristic not 2 such that $s^{n(s)}=s$ for all $s \in S$. Let $J(R)$ be the radical of $R$. Then

(1) $R / J(R)$ is a subdirect sum of fields and $2 \times 2$ matrix rings over fields;

(2) $J(R)^{3}=(0)$;

(3) $R$ satisfies a polynomial identity of degree 8;

(4) $R$ is algebraic over $F$; in fact, $R$ is locally finite.

Proof. (1) and (2) follow immediately from Lemmas 3 and 4. Since $R / J(R)$ satisfies the identities of a $2 \times 2$ matrix ring, it satisfies a polynomial identity of degree 4 ; since $x \in J(R)$ implies $x^{2}=0, R$ must satisfy an identity of degree 8 .

Since the symmetric elements of $R$ are algebraic over $F$, we may apply a theorem of Baxter and Martindale [1] to see that $R$ is algebraic and locally finite.

Some open questions remain. First of all, are Theorems 1 and 2 valid for the characteristic 2 case? Clearly new methods would be needed for their proofs as Osborn's theorems depend on characteristic not 2 .

Secondly, does the analog of Theorems 1 and 2 hold if the condition is imposed on the skew elements instead? This has been shown to be true in [4] if $R$ is a division ring.

\section{REFERENCES}

1. W. E. Baxter and W. S. Martindale III, Rings with involution and polynomial identities, Canad. J. Math. 20 (1968), 465-473. MR 36 \#5168.

2. I. N. Herstein, Special simple rings with involution, J. Algebra 6 (1967), 369-375. MR 35 \#1633.

3. - Topics in ring theory, Univ. of Chicago Press, Chicago, Ill., 1969.

4. I. N. Herstein and S. Montgomery, $A$ note on division rings with involutions, Michigan Math. J. 18 (1971), 75-79.

5. N. Jacobson, Structure of rings, Amer. Math. Soc. Colloq. Publ., vol. 37, Amer. Math. Soc., Providence, R.I., 1956. MR 18, 373.

6. W. S. Martindale III, Jordan homomorphisms of the symmetric elements of a ring with involution, J. Algebra 5 (1967), 241.

7. — Rings with involution and polynomial identities, J. Algebra 11 (1969), 186-194.

8. J. M. Osborn, Jordan algebras of capacity two, Proc. Nat. Acad. Sci. U.S.A. 57 (1967), 582-588. MR 35 \#6727.

9. - (to appear).

De Paul University, Chicago, Illinois 60614

University of Southern California, Los Angeles, California 90007 